\title{
New pre-main sequence candidates in the Taurus-Auriga star forming region ${ }^{\star}$
}

\author{
L. Scelsi ${ }^{1}$, A. Maggio ${ }^{2}$, G. Micela ${ }^{2}$, I. Pillitteri ${ }^{2}$, B. Stelzer ${ }^{2}$, K. Briggs ${ }^{3}$, M. Güdel ${ }^{3}$, N. Grosso ${ }^{4}$, \\ M. Audard ${ }^{5}$, and F. Palla ${ }^{6}$
}

1 Dipartimento di Scienze Fisiche ed Astronomiche, Sezione di Astronomia, Università di Palermo, Piazza del Parlamento 1, 90134 Palermo, Italy e-mail: scelsi@oapa.astropa.unipa.it

2 INAF - Osservatorio Astronomico di Palermo, Piazza del Parlamento 1, 90134 Palermo, Italy

Paul Scherrer Institut, Würenlingen and Villigen, 5232 Villigen PSI, Switzerland

4 Laboratoire d'Astrophysique de Grenoble, Université Joseph-Fourier, 414 rue de la Piscine, 38041 Grenoble Cedex, France

Columbia Astrophysics Laboratory, Mail Code 5247, 550 West 120th Street, New York, NY 10027, USA

${ }^{6}$ INAF - Osservatorio Astrofisico di Arcetri, Largo E. Fermi 5, 50125 Firenze, Italy

Received 4 May 2006 / Accepted 3 Augut 2006

ABSTRACT

\begin{abstract}
Aims. We have studied the X-ray source population of the Taurus Molecular Cloud (TMC) to search for new members of the TaurusAuriga star forming region.

Methods. Candidate members have been selected among the X-ray sources detected in 24 fields of the XMM-Newton Extended Survey of the Taurus Molecular Cloud, having an IR counterpart in the 2MASS catalog, based on color-magnitude and color-color diagrams. Their X-ray spectral properties have been compared with those of known members and other X-ray sources in the same fields but without a NIR counterpart. A search for flare-like variability in the time series of all new candidates and the analysis of the $\mathrm{X}$-ray spectra of the brightest candidates have been used to identify sources with a high probability of membership.

Results. We have found that 347 of 1909 detected X-ray sources have an infrared counterpart in the 2MASS catalogue. Among them, we have selected 57 sources that are consistent with being new pre-main sequence star candidates at the distance of the Taurus-Auriga star forming region; the X-ray spectral properties of this sample are, on the whole, similar to the properties of known TMC members and different from those of X-ray sources without an IR counterpart, most of which are likely to be of extragalactic origin. For 12 such candidates, the likelihood of membership is very high, based on the relatively high plasma temperatures derived from their X-ray spectra and/or the observation of powerful flares in their light curves.
\end{abstract}

Key words. X-rays: stars - Galaxy: open clusters and associations: individual: Taurus Molecular Cloud - stars: activity stars: coronae - stars: pre-main-sequence - stars: luminosity function, mass function

\section{Introduction}

Stellar population studies in star forming regions (SFR) are of fundamental importance to address issues related to the formation of stars and planets and the evolution of stellar properties during the early stages of their life. These issues include the determination of the stellar Initial Mass Function (IMF), which is an important test for theories of fragmentation and gravitational collapse of molecular clouds that lead to star formation, as well as the study of the strong magnetic activity (and its early evolution) responsible for the high levels of X-ray emission in young low-mass stars.

In this context, X-ray observations have proven to be an important method to discover new pre-main sequence stars. Owing to their intense X-ray emission, from 10 to $10^{4}$ times the solar level (e.g. Feigelson \& Montmerle 1999; Stelzer \& Neuhäuser 2001; Ozawa et al. 2005), and to the relatively low interstellar absorption at these wavelengths, X-ray observations are particularly efficient in detecting the population of young objects,

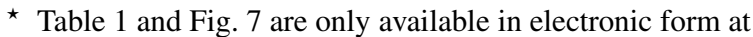
http://www . aanda. org namely classical T Tauri stars (CTTs), i.e. stars still accreting material from a circumstellar disk, and "weak-lined" $\mathrm{T}$ Tauri stars (WTTs), where accretion has ended and whose disks are thin or even absent (Walter et al. 1988). Optical and infrared surveys have so far identified many classical and "weak-lined" T Tauri stars, although they may favour the detection of CTTs, because of their strong $\mathrm{H} \alpha$ emission and IR excess. X-ray observations can serve as a complement to optical/IR searches for new young stars, especially those of very low mass because they are more easily detected at X-ray wavelengths. Moreover, since the WTTs are generally less absorbed than CTTs, the former are expected to be more efficiently selected in X-ray surveys, thus helping to reduce the bias mentioned above.

The detection of WTTs is also particularly important to estimate the fraction of stars at a certain age and in a certain environment having a circumstellar disk, and hence to estimate the disk life times. This information is fundamental to understanding the evolution of the angular momentum during the earlier phases of the stellar life, as well as for studies on the formation of planetary systems. In the nineties, several ROSAT studies (e.g., Neuhäuser et al. 1995; Sterzik et al. 1995; Alcala et al. 1995) 
detected a widely dispersed stellar population in Taurus, but also around other star-forming regions. These stars may have drifted away from their location of formation, or have been formed at their present location while the molecular clouds have dispersed. However, their status as weak-lined T Tauri stars has been debated, based on arguments that favor ages beyond the ZAMS age (e.g., Briceño et al. 1997).

The Taurus Molecular Cloud (TMC) is one of the nearest star forming regions $(d \sim 140 \mathrm{pc})$ and covers a large portion of the sky ( $\sim 100$ square degrees); the currently known members $(\sim 350)$ of this cloud complex are spatially distributed with relatively low density $\left(1-10 \mathrm{pc}^{-3}\right)$ and their ages range typically from 1 to $10 \mathrm{Myr}$. The stellar mass function of this star forming region has been investigated by Briceño et al. (2002), Luhman et al. (2003a) and Luhman (2004), and recently updated by Guieu et al. (2006) with the identification of 5 new very lowmass stars and 12 brown dwarfs; it appears different from the IMF of denser regions, such as Orion and IC 348 (Muench et al. 2002; Luhman et al. 2003b, respectively), suggesting a possible dependence of the shape of the IMF on the environment. The Taurus IMF shows a peak at higher masses $\left(\sim 0.8 M_{\odot}\right.$ vs. $\sim 0.2 M_{\odot}$ in Orion and IC 348), very few stars more massive than $\sim 1 M_{\odot}$ and a rather flat low-mass tail. A possible interpretation of this unusual IMF has recently been given in terms of core collapse and fragmentation, jointly with the ejection of very lowand substellar-mass embryos (Goodwin et al. 2004). However, more complete studies of the Taurus population are needed to assess the shape of the IMF with greater confidence, especially at the low-mass end.

The densest regions of Taurus have been surveyed by XMM-Newton, thus allowing a detailed study of the X-ray emission from young objects of this SFR. In particular, XMM-Newton has recently observed 19 regions, with a circular field of view with a diameter of $\sim 30$ arcmin and an exposure time of $\sim 30 \mathrm{ks}$ each; together with 8 more fields in the archive, these observations are the basis for the XMM-Newton Extended Survey of the Taurus Molecular Cloud (XEST), presented in detail in Güdel et al. (2007a). The total surveyed area covers about 5 square degrees of the TMC and contains about 150 known members, mainly classical and "weak-lined" T Tauri stars, but also protostars, brown dwarfs and a few Herbig Ae/Be stars and other members with uncertain classification.

This work focuses on the search for new pre-main sequence candidates among the X-ray detected sources in the fields of this XMM-Newton survey, also employing the near infrared data from the 2MASS point source catalogue (Skrutskie et al. 2006). The paper is organized as follows: Sect. 2 summarizes the main information about the observations analyzed here, the data reduction and the source detection procedure; in Sect. 3 we describe the method used to identify potential new TMC members and present the list of such candidates, while we discuss the global X-ray properties of different samples of XEST sources in Sect. 4. We discuss the temporal variability of all newly identified candidates and the analysis of the EPIC PN spectra of the brightest candidates in Sect. 5. In Sect. 6 we discuss the possible implications of our findings on the Taurus IMF. Our results are summarized in Sect. 7.

\section{Observations and source detection}

The present work is based on X-ray data taken with the European Photon Image Camera (EPIC MOS and EPIC PN, Turner et al. 2001; Strüder et al. 2001) located in the focal plane of the $\mathrm{X}$-ray telescopes on board XMM-Newton. These non-dispersive
CCD detectors, with spectral resolution $R=E / \Delta E \sim 5-50$ in the range $0.1-10 \mathrm{keV}$, and the mirrors provide a spatial resolution of $\sim 4-5^{\prime \prime}$ and effective areas of $\sim 1200 \mathrm{~cm}^{2}$ (PN) and $\sim 400 \mathrm{~cm}^{2}$ (MOS1 and MOS2) at $1.5 \mathrm{keV}$.

Among the observations analyzed in this study, the new ones (fields XEST-02 to XEST-20) were obtained in two separate periods (August-September 2004 and February-March 2005) and have PN exposure times ranging from 27 to $35 \mathrm{ks}$, while the five fields ${ }^{1}$ in the archive were observed for longer times (35-63 ks, see below the case of XEST-26). For all fields of the XMM-Newton survey of Taurus we have data from all three EPIC instruments, except for the field XEST-26 (around SU Aur) which lacks the PN data (the MOS exposures for this observation are $\sim 127 \mathrm{ks}$ ). All PN and MOS cameras were operated in full window mode, except for the field XEST-20 (around V773 Tau) where MOS2 was operated in small window mode, and the medium filter was applied in all observations, with the exception of the fields XEST-26 and XEST-27 (around $\zeta$ Per) where the thick filter was used. All observations were processed using SAS v6.1. We refer to the paper by Güdel et al. (2007a) for more details about the observations and the data reduction. The same paper gives also details relevant to the procedure used to detect X-ray sources in the EPIC images. In brief, source detection was performed on the sum of PN, MOS1 and MOS2 images, in three different bands, i.e.full $(0.5-7.3 \mathrm{keV})$, soft $(0.5-2 \mathrm{keV})$ and hard $(2-7.3 \mathrm{keV})$ band; the choice of these bands was mainly based on considerations on the expected spectra of the sources of interest and on the energies of strong features in typical EPIC background spectra. Time filtering of the images, yielding the Good Time Intervals (GTI), was applied to remove time ranges affected by high level of background emission and to maximize the signal-to-noise ratio of weak sources. Two source detection algorithms were used in sequence: the PWXDETECT method by Damiani et al. (1997), based on the wavelet transform of the X-ray image, was employed to locate source candidates which were confirmed or rejected afterwards by a maximum likelihood fitting of the spatially-dependent PSF using the SAS task EMLDETECT. The whole procedure was calibrated through extensive simulations so as to give one expected spurious detection per field due to fluctuations of the background. Moreover, clearly spurious sources were removed, such as detections in the diffraction features of bright sources. Typically, the number of X-ray sources in each field is between 50 and 100; in total, the number of detected sources from this survey is about 2000. The detection limit in each field depends on the off-axis angle and on the exposure time obtained after excluding the time intervals of high background radiation; for a typical observation with an average background contamination level, the X-ray luminosity detection threshold (at the distance of the Taurus Molecular Cloud) is $\approx 9 \times 10^{27} \mathrm{erg} \mathrm{s}^{-1}$ on-axis and $\approx 1.3 \times$ $10^{28} \mathrm{erg} \mathrm{s}^{-1}$ at $10^{\prime}$ off-axis for an X-ray source with a characteristic thermal spectrum having average temperature of the order of $\sim 10 \mathrm{MK}$ and hydrogen column density $N_{\mathrm{H}}=3 \times 10^{21} \mathrm{~cm}^{-2}$. Most of the detected sources have no infrared counterpart in the 2MASS catalogue and are likely of extragalactic origin (see Sect. 4), while many of the X-ray sources with IR counterpart lack any identification in the SIMBAD database. We detected

\footnotetext{
1 The field L1495 around V410 Tau was observed twice; the two observations are labelled XEST-23 and XEST-24. The fields around T Tau, AA Tau and BP Tau (XEST-01, XEST-25 and XEST-28, respectively) were not used; these are outlying fields, containing only one known TMC member each in the cases of XEST-25 and XEST-28, and three known members, but far away from the rest of TMC, in the case of XEST-01.
} 
121 of 153 known TMC members in the analyzed fields; their $\mathrm{X}$-ray properties are discussed in companion papers by Güdel et al. (2007b); Briggs et al. (2007); Grosso et al. (2007a,b); Stelzer et al. (2007); Telleschi et al. (2007a,b); Franciosini et al. (2007); Audard et al. (2007), while in the present work they have been used for comparison purposes with the other sources.

\section{Selection of new TMC candidate members}

In order to find possible new members of the Taurus-Auriga star forming region among the XEST sources, we identified these X-ray sources with NIR counterparts in the 2MASS point source catalogue, and plotted their position in IR color-magnitude and color-color diagrams.

After correcting the X-ray source coordinates for systematic offsets in the boresight positions (Güdel et al. 2007a), we searched for possible IR counterparts to each X-ray source within a radius of $3^{\prime \prime}$ from its centre. This choice ensures $\lesssim 1$ false association per field and leads to $347 \mathrm{X}$-ray sources associated with a point-like source listed in the 2 MASS catalogue.

We used the tables reported in Güdel et al. (2007a) and the SIMBAD database to identify respectively known members of the TMC and other sources already known to be non-members of the TMC. At this stage, we considered all the sources with accurately measured IR photometry in the $J, H$ and $K_{\mathrm{s}}$ bands, i.e. we excluded a few 2 MASS sources with upper limits of at least one magnitude, or whose measurements are flagged as inaccurate in the catalogue because of contamination from extended sources or image artifacts. We report the positions of the selected sources in the $J$ vs. $J-K_{\mathrm{s}}$ and $H$ vs. $H-K_{\mathrm{s}}$ diagrams (Fig. 1) together with the isochrones at 1, 5 and $10 \mathrm{Myr}$ of Siess et al. (2000) and Baraffe et al. (1998), calculated for a distance of $140 \mathrm{pc}$. Assuming a geometrical extension of the molecular cloud of $\sim 15$ pc leads to a spread of the tracks within $\sim 0.25 \mathrm{mag}$. The $J, H$ and $K_{\mathrm{s}}$ magnitudes are not corrected for reddening. Figure 2 shows the $J-H$ vs. $H-K_{\mathrm{s}}$ diagram. We selected sources satisfying the following criteria: (i) position in the color-magnitude diagrams above or compatible with the evolutionary models at $10 \mathrm{Myr}$ within the error bars; (ii) $J$ and $H$ magnitudes brighter than 16.5 and 15.5 respectively, which allows us to also include possible moderately absorbed brown dwarfs (the evolutionary models of Baraffe et al. extend down to $0.02 M_{\odot}$ ) and, at the same time, to avoid significant contamination of the sample with background objects; (iii) for sources lying on (or close to) the evolutionary models in the color-magnitude diagrams, that would indicate low absorption if they are members of this SFR, the same indication of low absorption must also be present in the color-color diagram.

The sample of photometric candidate members selected in this way contains 57 XEST sources reported in Table 1. Figures 1 and 2 show that most of the candidates tend to occupy the portions of the diagrams indicating lower absorption (or optically thinner disks) with respect to the known members, as we could expect from a WTTs-dominated population. From Fig. 2 we also note that only two candidates (XEST-02005 and XEST-06-045) show a clear IR excess, since they lie outside the region of the color-color diagram where stars with no excess reddening are expected to be. However, several classical T Tauri stars ${ }^{2}$ of the TMC are located within this region (Güdel et al. 2007a), hence we can not rule out that new CTTs may be found among our candidates.

${ }^{2}$ About half of the 121 detected known TMC members are protostars and CTTs.
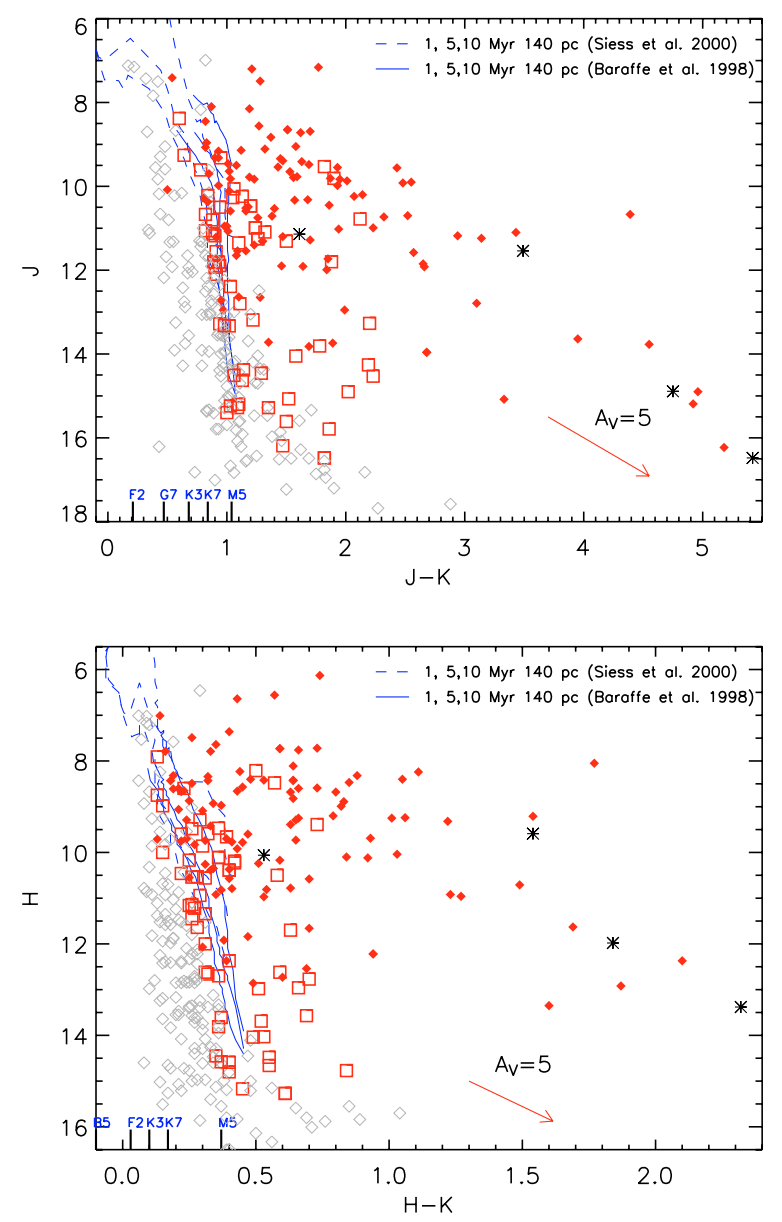

Fig. 1. Infrared color-magnitude diagrams with the observed (i.e. not dereddened) positions of the IR counterparts to the XEST X-ray sources. Known TMC members are shown as filled diamonds and asterisks (asterisks are protostars), candidate members as open squares and sources that are not selected as new candidates as open diamonds. Isochrones of 1, 5 and $10 \mathrm{Myr}$ (solid and dashed lines), calculated at $140 \mathrm{pc}$, are superimposed. The reddening vectors from the extinction laws by Rieke \& Lebofsky (1985) are also shown.

Table 1 contains information about the main X-ray and IR properties of our candidates. Note that four candidates (XEST-23-065, XEST-03-033, XEST-08-033 and XEST-08-049) are reported twice in the table with different XEST names, since they were detected by XMM-Newton in two overlapping fields. X-ray count-rates (CR) are on-axis equivalent rates for the $\mathrm{PN}$ in the $0.5-7.3 \mathrm{keV}$ band, averaged over the entire observation; for the sources XEST-03-028, XEST-07-005, XEST-17-043, XEST-27-084 and XEST-08-003, that show a quiescent phase preceeded or followed by a strong flare (see Sect. 5), we report also the values representing the quiescent level. The X-ray luminosities, in the $0.3-10 \mathrm{keV}$ band, were estimated from count-rates as follows. Using data relevant to the known TMC members surveyed by XMM-Newton (Güdel et al. 2007a), $L_{X}$ was found to be proportional to the PN on-axis count-rate with the conversion law:

$L_{\mathrm{X}, 30}=\left(a N_{\mathrm{H}, 22}+b\right) C R, \quad a=19.8 \pm 1.6, \quad b=4.2 \pm 0.7$

where $L_{\mathrm{X}, 30}$ is in units of $10^{30} \mathrm{erg} \mathrm{s}^{-1}$ and $N_{\mathrm{H}, 22}$ in units of $10^{22} \mathrm{~cm}^{-2}$.

The same law was assumed for our sample of candidates, for which the $N_{\mathrm{H}}$ was estimated using approximate visual 


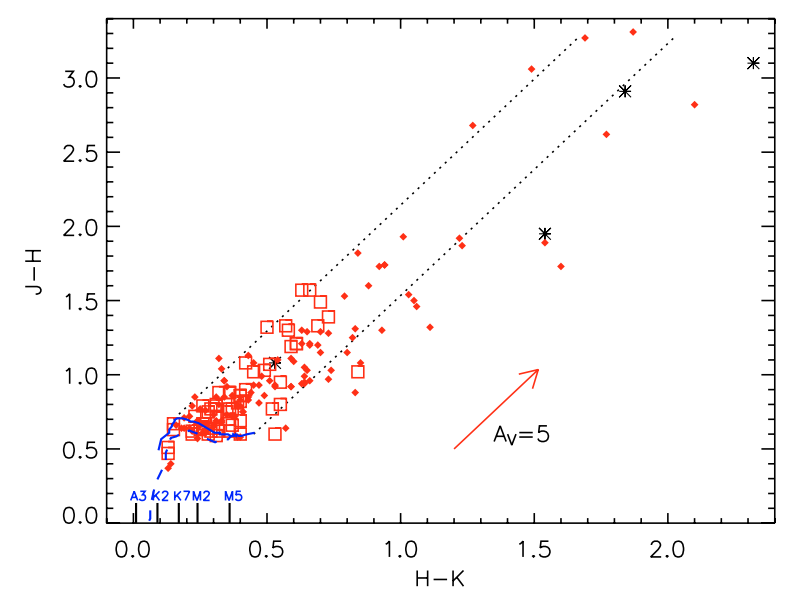

Fig. 2. Infrared color-color diagram with the observed (i.e. not dereddened) positions of known TMC members and candidate members (symbols are as in Fig. 1). The solid and dashed lines are the $10 \mathrm{Myr}$ models by Baraffe et al. (1998) and Siess et al. (2000) respectively. The reddening vector is shown. The dotted lines bracket the region where stars with no excess reddening are expected to lie.

absorptions from the color-color diagram ${ }^{3}$ and the relation for the interstellar medium between $N_{\mathrm{H}}$ and $A_{\mathrm{V}}$. In our Galaxy the ratio $N_{\mathrm{H}} / A_{\mathrm{V}}$ is found in the range $1.8-2.2 \times 10^{21} \mathrm{~cm}^{-2}$ (Ryter et al. 1975; Gorenstein 1975; Bohlin et al. 1978; Whittet 1981; Predehl \& Schmitt 1995; Ryter 1996); we used the conversion $N_{\mathrm{H}}=2 A_{\mathrm{V}} \times 10^{21} \mathrm{~cm}^{-2}$. For the 5 flaring sources mentioned above, $L_{\mathrm{X}}$ refers to the quiescent emission. For $9 \mathrm{bright}$ X-ray candidates (XEST-09-042, XEST-08-049, XEST-20-071, XEST-08-014, XEST-09-033, XEST-08-033, XEST-17-059, XEST-05-027 and XEST-15-034) we report the luminosities derived directly from spectral fitting of the PN data (Sect. 5, Table 2); we have verified that in these cases the luminosities are in good agreement with the values obtained from converting count-rates into $L_{X}$. Individual errors on $L_{X}$ are not reported, due to the approximations of the relationship above, the uncertainty on the estimated $A_{\mathrm{V}}$ and to the intrinsical variability of $\mathrm{T}$ Tauri stars (typically within a factor of 2). Overall, the X-ray luminosities are uncertain by $0.2-0.3$ dex.

\section{Analysis of X-ray spectral properties}

In this section we compare the X-ray spectral properties of our TMC candidates with those of known members and X-ray sources without IR counterparts. For this study, we used the EPIC PN data in the $0.5-7.3 \mathrm{keV}$ band. Source events were extracted from circular regions whose extentions were calculated so as to maximize the source signal-to-noise ratio and to exclude the events of possible nearby contaminating sources: the extraction radii for the sources listed in Table 1 are in the range $\sim 10^{\prime \prime}-50^{\prime \prime}$. For the background photons, we used annular regions around the source and also excluded the events in elliptical regions of close sources overlapping within the annulus.

The analysis presented here has been conducted using diagrams analogous to color-color X-ray plots: in particular, we employed the quantile-based method proposed by Hong et al. (2004), which is useful when dealing with low-count sources,

\footnotetext{
3 Note that reasonable estimates of $A_{\mathrm{V}}$ are obtained from the color-color diagram if our sources show no significant IR excess; this is consistent with the finding that our sample could be dominated by WTTs.
}

as are most of ours. Instead of working with predefined energy bands to derive hardness-ratios, a procedure which does not allow us to optimize the statistics for all sources at the same time ${ }^{4}$, we determined for each source spectrum the energy value that divides the detected photons in predefined fractions of the total counts. In particular, following Hong et al., we used the median $(50 \%)$ and the quartiles $(25 \%$ and $75 \%)$ to define the quantile $Q_{50}=\left(E_{50 \%}-E_{\min }\right) /\left(E_{\max }-E_{\min }\right)$ (with $E_{\max }=$ $7.3 \mathrm{keV}$ and $E_{\min }=0.5 \mathrm{keV}$ ), and analogous expressions for $Q_{25}$ and $Q_{75}$.

We report in the phase space $Q_{25} / Q_{75}$ vs. $Q_{50} /\left(1-Q_{50}\right)$ of Fig. 3 the positions of known TMC members and candidates (left panel) and of the X-ray sources without an IR counterpart (right panel). A theoretical grid predicted by an absorbed onecomponent coronal model, with fixed abundances $(0.3$ times the solar values of Anders \& Grevesse 1989) and different temperatures (going from $\log T=6.5$ up to $\log T=7.75$ ) and hydrogen column densities (from $N_{\mathrm{H}}=10^{20} \mathrm{~cm}^{-2}$ to $10^{23} \mathrm{~cm}^{-2}$ ), is superimposed. The degeneracy around $(X, Y)=(-1.2,0.4)$ expresses the similarity between moderately hot spectra with little absorption and cooler absorbed ones (for details about these topics, see also the appendix in Grosso et al. 2007b). At high temperatures, the degeneracy is removed and the effect of absorption is to shift the model points towards the upper right part of the plot. Note, however, that in this discussion the theoretical grid served only as a reference to understand where sources with thermal spectra should be expected to lie, due to the simplicity of the model and the uncertainty on the computed quantities. In particular, care must be taken in deriving temperature and hydrogen columns from the positions of the sources with respect to the grid in such diagrams, especially for high-statistics sources, since active stars usually have more complex spectra that cannot be described by a single-component model.

The large spread of the points, above all for the X-ray sources without IR counterpart, is dominated by the large error bars for the faintest sources. Typical errors for faint sources with $\sim 30$ and $\sim 50$ counts are, respectively, \pm 0.25 and \pm 0.15 on both the quantities on the $X$ and $Y$ axes in Fig. 3. However, we clearly observe that the sources without IR counterparts have in general spectra harder than those of TMC members and candidates; this trend is more evident in Fig. 4, where only sources with more than 80 counts, and hence smaller errors, are plotted. The former sample (i.e. X-ray sources without IR counterpart) is very likely dominated by extragalactic objects, as suggested by the fact that power laws that describe the spectra of AGNs lie exactly in the portion of the phase space occupied by these sources.

We also note that the distributions of candidates and TMC members in this quantile space are indistinguishable at both statistics we have shown, while they are different from the distribution of the X-ray sources without IR counterpart. It is also interesting to note that the sub-sample of known TMC members lying in the portion of the grid with high temperatures and hydrogen columns (essentially defined by $\log _{10}\left[Q_{50} /\left(1-Q_{50}\right)\right]>$ -0.8 ) is composed of stars with high $A_{V}$ and/or IR excess, as shown in the color-magnitude diagram of Fig. 5, classified as classical T Tauri stars. The candidates found in this work mainly lie at $\log _{10}\left[Q_{50} /\left(1-Q_{50}\right)\right]<-0.8$, where the TMC members are both classical and weak-lined T Tauri stars, with a slight predominance of WTTs. This result, together with the color-magnitude diagram of Fig. 1, suggests that our sample of candidates may be dominated by WTTs.

4 The counts in each band depend on the spectral shape of the source, which is in general different for the various sources. 

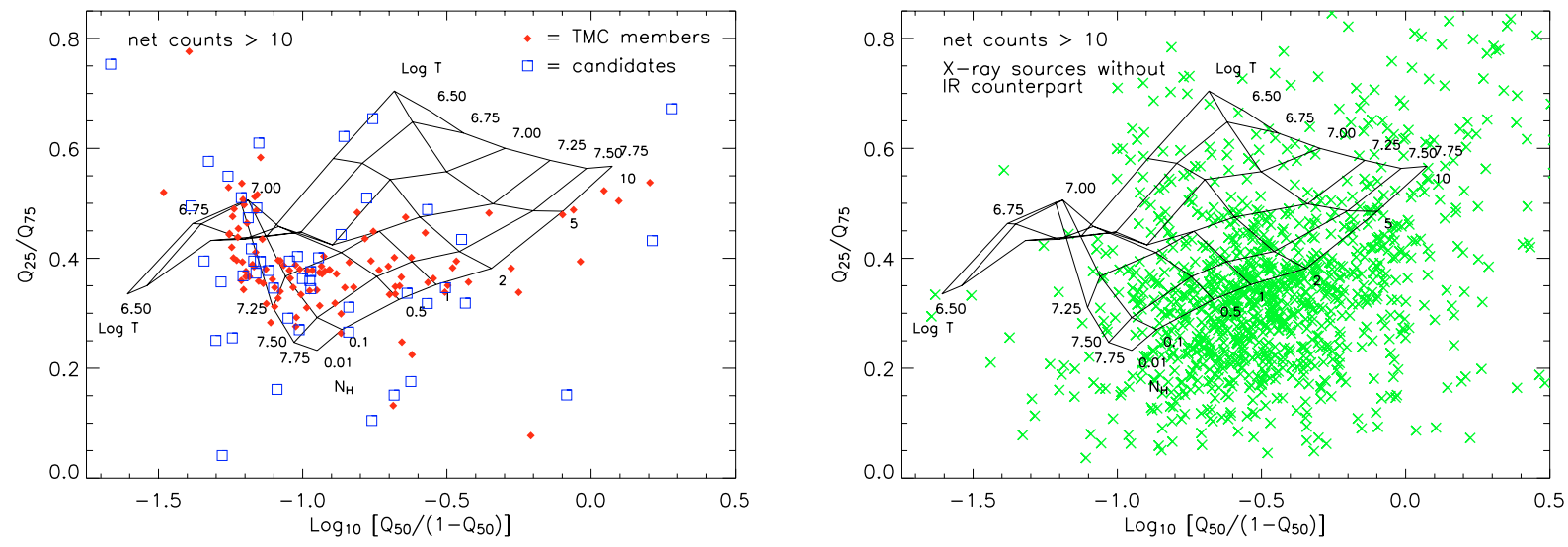

Fig. 3. Quantile space for X-ray sources with more than 10 net counts in the PN: known members (filled diamonds) and candidates (open squares) are shown in the left panel, X-ray sources without an IR counterpart are shown as crosses in the right panel. A theoretical grid relevant to an absorbed, isothermal and optically thin plasma is superimposed, with abundances fixed to 0.3 times the solar values of Anders \& Grevesse (1989), calculated for temperatures going from $\log T=6.5$ up to $\log T=7.75$ at a $\operatorname{logarithmic}$ step of 0.25 and seven values for the hydrogen column density $\left(N_{\mathrm{H}}=0.01,0.1,0.5,1,2,5\right.$ and 10 in units of $\left.10^{22} \mathrm{~cm}^{-2}\right)$.
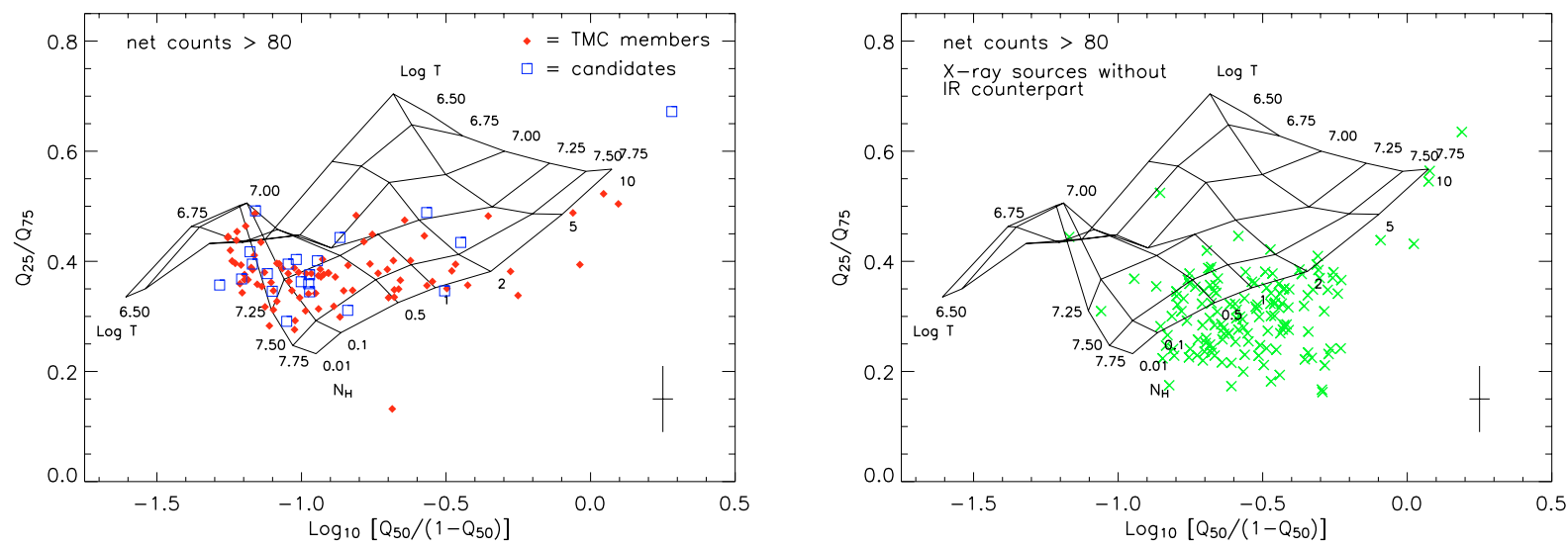

Fig. 4. Same as the previous figure, but for sources with more than 80 net counts in the PN. The error bars are generally smaller than those shown in the lower right part of the plots, typical of sources with about 80 counts.

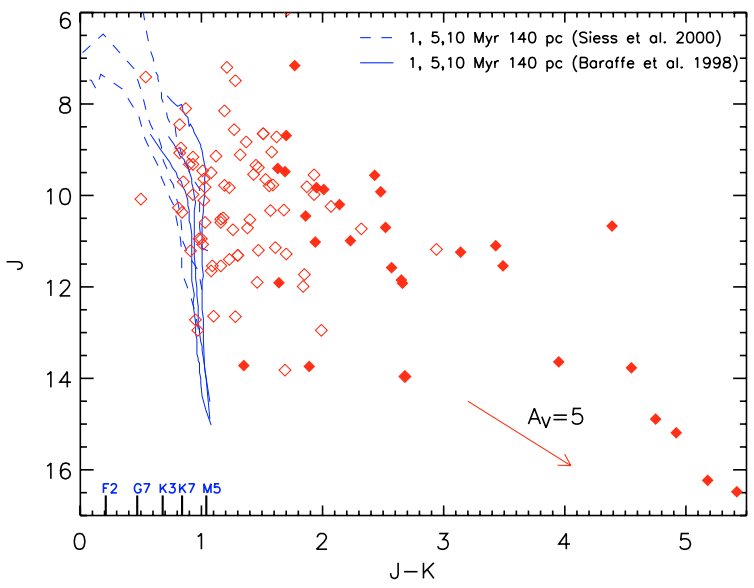

Fig. 5. IR color-magnitude diagram for the TMC members shown in the left panel of Fig. 3: filled and open diamonds refer to stars lying, in the quantile space, at $\log _{10}\left[Q_{50} /\left(1-Q_{50}\right)\right]$ greater and lower than -0.8 , respectively.

\section{Candidates with high probability of membership}

Since young stellar objects frequently show variability of their $\mathrm{X}$-ray emission on short ( ks) time scales and also exhibit powerful explosive events (Stelzer et al. 2000; Imanishi et al. 2001), we analysed the photon time-series of all candidates in Table 1 to search for variability, and in particular the presence of flare-like features, supporting the coronal origin and youth of these sources. We used a maximum likelihood algorithm (MLB) that searches for variability in unbinned series of photon arrival times. Details of the method and its application to XEST data are described by Stelzer et al. (2007); it is similar to the Scargle method (Scargle 1998), but is based on a maximum likelihood procedure instead of the Bayesian approach. In brief, the algorithm identifies time intervals of constant signal in photon time series under the assumption of Poisson noise. Background subtraction is done on the photon events list individually for each source before the algorithm is applied. The resulting "sourceonly" light curve consists of segments with different intensity levels. The technique has two free parameters, the confidence level for the intensity changes and the minimum number of counts $\left(N_{\min }\right)$ that define a segment. Small numbers for $N_{\min }$ facilitate the detection of variability in faint sources, but have also a tendency to find variations that are likely spurious. Stelzer et al. (2007) find in their variability analysis of known TMC members that $N_{\min }=20$ is an adequate compromise.

This method indicates that 16 of the 57 XEST sources identified as candidate TMC members are variable in the broad band $(0.3-7.8 \mathrm{keV})$ at the $99 \%$ level; they are flagged in 

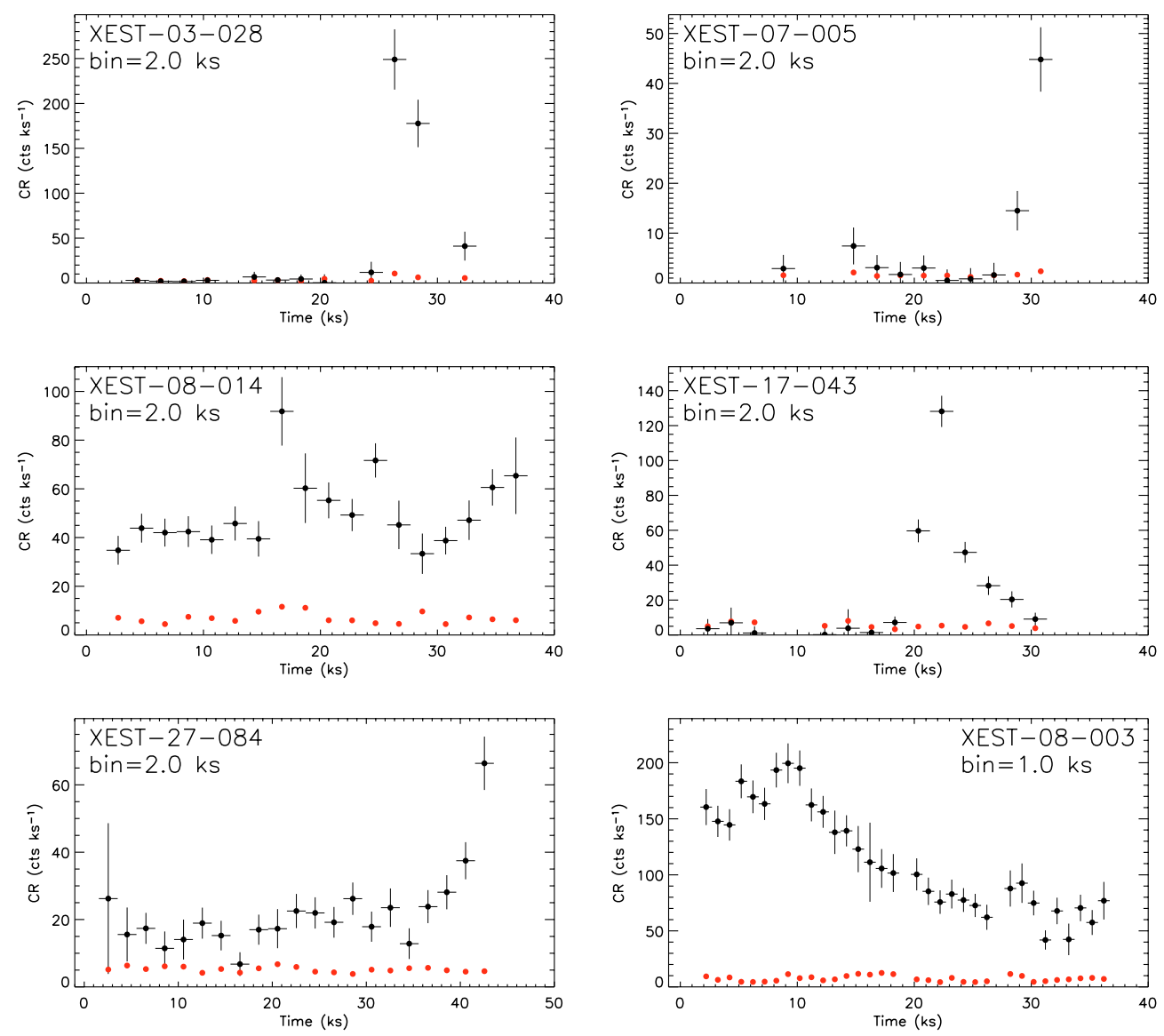

Fig. 6. Background-subtracted light curves (points with error bars) of six sources showing flares, obtained adding the PN, MOS1 and MOS2 counts. The points without errors represent the background emission.

Table 1. Among them, the sources XEST-03-028, XEST-07-005, XEST-08-014, XEST-17-043, XEST-27-084 and XEST-08-003 show intense flares in their light curves (Fig. 6). We conclude that these 6 sources have higher probability of resulting true members of the Taurus Molecular Cloud.

Eleven XEST sources among the candidates listed in Table 1 have enough $(\gtrsim 1000)$ net counts in the PN camera to allow global fits of their spectra. Three of them are the flaring sources XEST-08-003, XEST-08-014 and XEST-27-084 mentioned above, while XEST-08-049 and XEST-09-033 are identified with the same 2MASS object. After the response functions were built with the SAS tasks arfgen and rmfgen, we tried to fit in XSPEC the spectra of these sources using both an absorbed power law and an absorbed, optically thin plasma based on the APEC code (Smith et al. 2001), with one (or two) isothermal component(s). The abundances of the coronal models were fixed at values typical for pre-main sequence or extremely active stars, based on results from Argiroffi et al. (2004); García-Alvarez et al. (2005); Scelsi et al. (2005); Telleschi et al. $(2005)^{5}$. We found that all these spectra are well described by coronal models (Fig. 7) but not by power laws (except the source XEST-05-027 discussed below), thus excluding an extragalactic nature. The parameters of the best-fit models are listed in Table 2. The model temperatures are relatively (or very) high and characteristic of

5 With respect to the solar photospheric values by Anders \& Grevesse (1989), the adopted abundances are: $\mathrm{C}=0.45, \mathrm{~N}=0.788, \mathrm{O}=0.426$, $\mathrm{Ne}=0.832, \mathrm{Mg}=0.263, \mathrm{Al}=0.5, \mathrm{Si}=0.309, \mathrm{~S}=0.417, \mathrm{Ar}=0.55$, $\mathrm{Ca}=0.195, \mathrm{Fe}=0.195, \mathrm{Ni}=0.195$. The same set of abundances was used by Güdel et al. (2007a) to fit the spectra of known TMC members. young active stars (e.g. Preibisch et al. 2005); the X-ray luminosities derived from the measured unabsorbed flux and assuming a distance of $140 \mathrm{pc}$ are in the range $10^{29}-3 \times 10^{30} \mathrm{erg} \mathrm{s}^{-1}$, also typical of active stars (Stelzer \& Neuhäuser 2001; Preibisch et al. 2005). Therefore, based on these considerations, we conclude that all these sources have a higher probability of being true TMC members.

The photon spectrum of XEST-05-027 can also be described by an absorbed power law which provides $\chi_{v}^{2}=0.80$ (17 d.o.f.) and thus formally a better fit than the coronal model. The derived hydrogen column density $\left(\sim 1.3 \times 10^{22} \mathrm{~cm}^{-2}\right)$ and power law in$\operatorname{dex}(\sim-2.2)$ are values typically found for AGNs (e.g. Hasinger et al. 2001), yet the infrared magnitudes of the IR counterpart would be particularly bright for an extragalactic source, and this makes us confident that the nature of this source is more likely a stellar one. The average temperature of the coronal model is very high $(\sim 48 \mathrm{MK})$, but the light curve is consistent with a constant emission, as confirmed by both the MLB and the Kolmogorov-Smirnov tests. So, although we cannot completely rule out an extragalactic origin, this heavily absorbed source deserves attention in the future. An alternative possibility is that a chance association between a stellar IR source and an X-ray extragalactic source occurred in this case ${ }^{6}$.

Most of the candidates with a higher probability of membership (8 of 12) are located in three adjacent fields: XEST-08, XEST-09 and XEST-17 (for a map of the TMC and the

${ }^{6}$ We expect at most about 20 false associations among the 226 (i.e. 347 minus 121 TMC members) X-ray sources with an IR counterpart within $3^{\prime \prime}$. 
Table 2. Parameters of the best-fit coronal models (with $1 \sigma$ errors) for the brightest candidate members. $T_{\text {av }}$ is the EM-weighted logarithmic average temperature, defined as $\log T_{\mathrm{av}}=\left(E M_{1} \log T_{1}+E M_{2} \log T_{2}\right) /\left(E M_{1}+E M_{2}\right)$ (see also Güdel et al. 2007a). The model flux (in the $0.3-10 \mathrm{keV}$ band ) is unabsorbed and the luminosity is calculated from it assuming $d=140 \mathrm{pc}$.

\begin{tabular}{|c|c|c|c|c|c|c|c|c|c|}
\hline Source & $\begin{array}{c}N_{\mathrm{H}} \\
\left(10^{22} \mathrm{~cm}^{-2}\right)\end{array}$ & $\begin{array}{c}T_{1} \\
(\mathrm{MK})\end{array}$ & $\begin{array}{c}T_{2} \\
(\mathrm{MK})\end{array}$ & $E M_{2} / E M_{1}$ & $\begin{array}{c}T_{\mathrm{av}} \\
(\mathrm{MK})\end{array}$ & $\begin{array}{c}F_{\mathrm{X}} \\
\left(10^{-13} \mathrm{cgs}\right)\end{array}$ & $\begin{array}{c}L_{\mathrm{X}} \\
\left(10^{28} \mathrm{cgs}\right)\end{array}$ & $\chi_{v}^{2}$ (d.o.f.) & Flare \\
\hline XEST-09-042 & $0.12 \pm 0.01$ & $7.9 \pm 0.3$ & $20 \pm 2$ & $0.71 \pm 0.07$ & 11 & 7.3 & 172 & $0.95(147)$ & \\
\hline XEST-08-049 ${ }^{a}$ & $0.30 \pm 0.02$ & $9.2 \pm 0.6$ & $23 \pm 2$ & $1.4 \pm 0.3$ & 16 & 4.9 & 115 & $1.29(86)$ & \\
\hline XEST-08-003 & $0.24 \pm 0.02$ & $8.9 \pm 0.7$ & $50 \pm 6$ & $2.8 \pm 0.6$ & 31 & 13 & 290 & $0.81(79)$ & yes \\
\hline XEST-20-071 & $0.41 \pm 0.04$ & $7.8 \pm 0.6$ & $18_{-2}^{+3}$ & $0.50 \pm 0.15$ & 10 & 7.3 & 171 & $0.74(49)$ & \\
\hline XEST-08-014 & $<0.02$ & $4.7 \pm 0.4$ & $17_{-2}^{+3}$ & $0.88 \pm 0.16$ & 8.6 & 1.3 & 30 & $1.50(30)$ & yes \\
\hline XEST-09-033 ${ }^{a}$ & $0.25 \pm 0.05$ & $14.5 \pm 0.8$ & - & - & 14.5 & 2.9 & 68 & $1.38(26)$ & \\
\hline XEST-08-033 & $0.40 \pm 0.10$ & $4.8_{-0.7}^{+1.8}$ & $16.1_{-1.7}^{+1.3}$ & $0.8 \pm 0.4$ & 8.3 & 1.6 & 38 & $1.47(22)$ & \\
\hline XEST-17-059 & $0.18 \pm 0.03$ & $13.0 \pm 0.6$ & -1.1 & - & 13 & 1.7 & 40 & 1.09 (27) & \\
\hline XEST-05-027 & $1.10 \pm 0.15$ & $48_{-11}^{+20}$ & - & - & 48 & 8.6 & 202 & 0.89 (17) & \\
\hline XEST-15-034 & $<0.02$ & $6.0 \pm 0.7$ & - & - & 6.0 & 0.42 & 10 & 0.78 (19) & \\
\hline XEST-27-084 & $0.07 \pm 0.04$ & $9.1 \pm 0.8$ & $33_{-9}^{+17}$ & $0.8 \pm 0.3$ & 17 & 0.57 & 13 & $0.67(21)$ & yes \\
\hline
\end{tabular}

${ }^{a}$ These two sources have the same 2MASS counterpart.

XEST fileds, see Fig. 1 in Güdel et al. 2007a); these fields do not seem to be special with respect to the others, and the density of known members is not particularly high in them. Hence, our finding may deserve further investigation.

\section{Discussion}

In this section we explore the possible implications of our candidate members on the Taurus IMF. To estimate the masses of the selected sources, we evaluated visual extintions $A_{\mathrm{V}}$ from the color-color diagram (Fig. 2), then we used these extintions to place the stars in Fig. 1 on the evolutionary tracks by Baraffe et al. (1998), and finally we searched for compatible values of mass and age in both color-magnitude diagrams (Fig. 1). Note, however, that these estimates may be affected by large uncertainties, due to the estimated values of $A_{\mathrm{V}}$, to the assumption of no IR excess and also to the uncertainties in the evolutionary models.

Consider first the sub-sample of candidates with available PN spectra. We noted that the visual extinctions obtained from the IR color-color diagram are consistent with those calculated from the hydrogen column densities using the conversion $N_{\mathrm{H}}=$ $2 A_{\mathrm{V}} \times 10^{21} \mathrm{~cm}^{-2}$ for all sources listed in Table 2, with the exception of XEST-20-071; we exclude this star from the following discussion together with the source XEST-05-027 because the two pairs of mass and age indicated by the two color-magnitude diagrams were largely incompatible. The general agreement between $A_{\mathrm{V}}$ and $N_{\mathrm{H}}$ implies no significant IR excess due to a circumstellar disk for these sources. After dereddening, their positions in the color-magnitude diagrams yield estimated masses in the range $0.2-0.6 M_{\odot}$ and ages ranging from $\sim 1$ to $\gtrsim 10 \mathrm{Myr}$, in agreement with those of known TMC members; this result implies that our bright, most probable members are not very lowmass stars, and hence the known difference between the shape of the Taurus IMF and those of Orion and IC 348 at very low masses (see, for example, Fig. 1 in Goodwin et al. 2004) will not be reduced.

Since the above sample includes only the X-ray brightest sources, it may not come as a surprise that they are relatively massive pre-main sequence candidates, because a correlation between mass and $L_{X}$ has been found in Taurus (Güdel et al. 2007a) and also in other star forming regions (e.g. Preibisch \& Zinnecker 2002; Preibisch et al. 2005, for the cases of IC 348 and Orion, respectively). Therefore, the major implications for

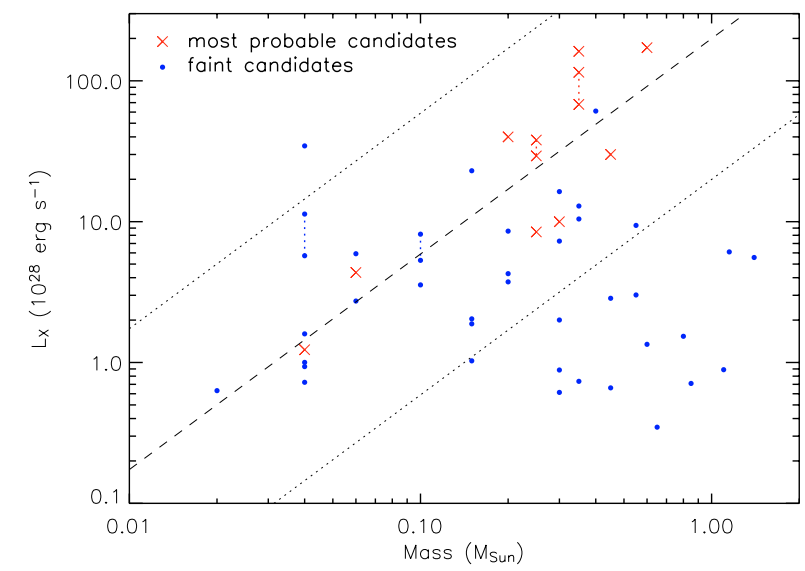

Fig. 8. X-ray luminosities versus masses for the 48 candidates with mass estimates. Crosses refer to the candidates with higher probability of membership. $L_{X}$ values joined by a dotted segment refer to luminosities derived for the same source in two different XEST observations. The dashed line is the relation $\log L_{\mathrm{X}}=1.53 \log M+30.3$ best-fitting the data for known TMC members (Güdel et al. 2007a) and the dotted lines bracket the region where known members are found.

the Taurus IMF could come from the faintest candidates. Since we have no estimates of $N_{\mathrm{H}}$ for these sources to be compared with the $A_{\mathrm{V}}$, we assumed that approximate $A_{\mathrm{V}}$ values can be derived from the color-color diagram and we used these estimates to deredden their positions in the color-magnitude diagrams. This procedure was applied to all the X-ray faint sources, except for XEST-02-005 which clearly shows IR excess and is therefore excluded. For 6 sources we could not obtain compatible mass and age values from both color-magnitude diagrams, and hence they are also excluded from the following discussion ${ }^{7}$. In total, we have 48 candidates (including the X-ray bright ones) with self-consistent extintions, mass and age estimates, which are plotted in Fig. 8: we observe that faint candidates span a relatively large mass range $\left(0.02-1.2 M_{\odot}\right)^{8}$ but they are not clustered

\footnotetext{
7 The candidate XEST-03-028 with high probability of membership is among the faint sources without mass estimate; its position in the IR diagrams might indicate very high absorption and/or IR excess.

8 The positions in the IR diagrams of the candidates XEST-07-005 and XEST-17-043 with high probability of membership could suggest for them a substellar mass.
} 
at very low masses. We also note that most of the candidates follow the $L_{\mathrm{X}}$-mass correlation derived for the other TMC members (Güdel et al. 2007a). That is independent support for these candidates being TMC members, and is especially true for the most probable candidates. Only those in the lower-right corner (all of them being "faint candidates") are suspiciously off.

In conclusion, our analysis suggest that the shape of the Taurus stellar IMF could not be affected significantly at the very low-mass end, even with the addition of all these candidates. However we recall that the masses derived in this section may be affected by large uncertainties; moreover it is quite possible that several candidates are not true TMC members (possibly the low-luminosity sources with apparently high mass, from Fig. 8), hence more detailed studies of these sources are required before any reliable assertion on the very low-mass end of the Taurus IMF can be stated.

\section{Conclusions}

As X-ray emission is particularly intense during the early stages of the stellar evolution, in this work we have employed X-ray data from the XEST survey and the 2MASS infrared data to identify possible new pre-main sequence stars belonging to the Taurus-Auriga star forming region. More precisely, in the 24 regions of the TMC observed by XMM-Newton and analyzed here, covering an area of $\sim 5$ square degrees, we identified $57 \mathrm{X}$-ray and IR sources compatible with being young stellar objects of this molecular cloud, and we found 12 sources among them with high probability of being TMC members based on the analysis of PN spectra and/or the presence of flares in the light curves. From the infrared color-magnitude and color-color diagrams, we note that most of these candidates could be weak-lined T Tauri stars that are less efficiently selected from population studies in the optical and infrared bands. If not TMC members, these sources might be foreground young main sequence stars or background active stars (M-type stars older than the Taurus population, binary RS CVn systems, bright single giants); the candidate sample might also include some extragalactic object among the faintest IR and X-ray sources; there is also a relatively small probability of chance associations between an IR stellar source and an extragalactic X-ray source. Optical follow-up observations aimed at collecting spectra for measurements of the $\mathrm{Li}$ $6708 \AA$ and $\mathrm{H} \alpha$ lines and radial velocities, and for spectral classification and effective temperatures determination, will be performed to determine the nature of the optical/IR counterparts for the X-ray sources identified in this work, and hence to confirm or reject their membership of the TMC; although we do not expect that all these sources will be confirmed as new members, these observations could lead to a significant increase of the number of WTTs in these surveyed fields, whose present number is 49 .

Acknowledgements. We acknowledge financial support by the International Space Science Institute (ISSI) in Bern to the XMM-Newton XEST team. The Palermo group acknowledges financial contribution from contract ASI-INAF I/023/05/0. X-ray astronomy research at PSI has been supported by the Swiss National Science Foundation (grants Nos. 2066875.01 and 20-109255/1). M.A. acknowledges support from NASA grant NNGO5GF92G. This research is based on observations obtained with XMM-Newton, an ESA science mission with instruments and contributions directly funded by ESA member states and the USA (NASA). This publication makes use of data products from the Two Micron All Sky Survey (2MASS), which is a joint project of the University of Massachusetts and the Infrared Processing and Analysis Center/California Institute of Technology, funded by the National Aeronautics and Space Administration and the National Science Foundation. Further, our research has made use of the SIMBAD database, operated at CDS, Strasbourg, France.

\section{References}

Alcala, J. M., Krautter, J., Schmitt, J. H. M. M., et al. 1995, A\&AS, 114, 109 Anders, E., \& Grevesse, N. 1989, Geochim. Cosmochim. Acta, 53, 197 Argiroffi, C., Drake, J. J., Maggio, A., et al. 2004, ApJ, 609, 925 Audard, M., Briggs, K. R., Grosso, N., et al. 2007, A\&A, 468, 379 Baraffe, I., Chabrier, G., Allard, F., \& Hauschildt, P. H. 1998, A\&A, 337, 403 Bohlin, R. C., Savage, B. D., \& Drake, J. F. 1978, ApJ, 224, 132 Briceño, C., Hartmann, L. W., Stauffer, J. R., et al. 1997, AJ, 113, 740 Briceño, C., Luhman, K. L., Hartmann, L., Stauffer, J. R., \& Kirkpatrick, J. D. 2002, ApJ, 580, 317

Briggs, K. R., Güdel, M., Telleschi, A., et al. 2007, A\&A, 468, 413 Damiani, F., Maggio, A., Micela, G., \& Sciortino, S. 1997, ApJ, 483, 350

Feigelson, E. D., \& Montmerle, T. 1999, ARA\&A, 37, 363

Franciosini, E., Pillitteri, I., Stelzer, B., et al. 2007, A\&A, 468, 485 Güdel, M., Briggs, K. R., Arzner, K., et al. 2007a, A\&A, 468, 353 Güdel, M., Telleschi, A., Audard, M., et al. 2007b, A\&A, 468, 515 García-Alvarez, D., Drake, J. J., Lin, L., Kashyap, V. L., \& Ball, B. 2005, ApJ, 621,1009

Goodwin, S. P., Whitworth, A. P., \& Ward-Thompson, D. 2004, A\&A, 419, 543 Gorenstein, P. 1975, ApJ, 198, 95

Grosso, N., Audard, M., Bouvier, J., Briggs, K. R., \& Güdel, M. 2007a, A\&A, 468,557

Grosso, N., Briggs, K. R., Güdel, M., et al. 2007b, A\&A, 468, 391

Guieu, S., Dougados, C., Monin, J.-L., Magnier, E., \& Martín, E. L. 2006, A\&A, 446,485

Hasinger, G., Altieri, B., Arnaud, M., et al. 2001, A\&A, 365, L45

Hong, J., Schlegel, E. M., \& Grindlay, J. E. 2004, ApJ, 614, 508

Imanishi, K., Koyama, K., \& Tsuboi, Y. 2001, ApJ, 557, 747

Luhman, K. L. 2004, ApJ, 617, 1216

Luhman, K. L., Briceño, C., Stauffer, J. R., et al. 2003a, ApJ, 590, 348

Luhman, K. L., Stauffer, J. R., Muench, A. A., et al. 2003b, ApJ, 593, 1093

Muench, A. A., Lada, E. A., Lada, C. J., \& Alves, J. 2002, ApJ, 573, 366

Neuhäuser, R., Sterzik, M. F., Torres, G., \& Martin, E. L. 1995, A\&A, 299, L13

Ozawa, H., Grosso, N., \& Montmerle, T. 2005, A\&A, 429, 963

Predehl, P., \& Schmitt, J. H. M. M. 1995, A\&A, 293, 889

Preibisch, T., \& Zinnecker, H. 2002, AJ, 123, 1613

Preibisch, T., Kim, Y.-C., Favata, F., et al. 2005, ApJS, 160, 401

Rieke, G. H., \& Lebofsky, M. J. 1985, ApJ, 288, 618

Ryter, C. E. 1996, Ap\&SS, 236, 285

Ryter, C., Cesarsky, C. J., \& Audouze, J. 1975, ApJ, 198, 103

Scargle, J. D. 1998, ApJ, 504, 405

Scelsi, L., Maggio, A., Peres, G., \& Pallavicini, R. 2005, A\&A, 432, 671

Siess, L., Dufour, E., \& Forestini, M. 2000, A\&A, 358, 593

Skrutskie, M. F., Cutri, R. M., Stiening, R., et al. 2006, AJ, 131, 1163

Smith, R. K., Brickhouse, N. S., Liedahl, D. A., \& Raymond, J. C. 2001, ApJ, 556, L91

Stelzer, B., \& Neuhäuser, R. 2001, A\&A, 377, 538

Stelzer, B., Neuhäuser, R., \& Hambaryan, V. 2000, A\&A, 356, 949

Stelzer, B., Flaccomio, E., Briggs, K. R., et al. 2007, A\&A, 468, 463

Sterzik, M. F., Alcala, J. M., Neuhaeuser, R., \& Schmitt, J. H. M. M. 1995, A\&A, 297, 418

Strüder, L., Briel, U., Dennerl, K., et al. 2001, A\&A, 365, L18

Telleschi, A., Güdel, M., Briggs, K. R., Audard, M., \& Scelsi, L. 2007a, A\&A, 468, 443

Telleschi, A., Güdel, M., Briggs, K. R., et al. 2007b, A\&A, 468, 541

Telleschi, A., Güdel, M., Briggs, K., et al. 2005, ApJ, 622, 653

Turner, M. J. L., Abbey, A., Arnaud, M., et al. 2001, A\&A, 365, L27

Walter, F. M., Brown, A., Mathieu, R. D., Myers, P. C., \& Vrba, F. J. 1988, AJ, 96, 297

Whittet, D. C. B. 1981, MNRAS, 196, 469 
L. Scelsi et al.: New pre-main sequence candidates in Taurus, Online Material $p 1$

\section{Online Material}


Table 1. List of all candidate members. "XEST id" refers to the name of the X-ray source in the XEST catalogue (Güdel et al. 2007a, the first 2 digits mark the field of the survey). RA $X$ and Dec $X$ are the coordinates of the $X$-ray source (corrected for boresight shift). CR is the equivalent on-axis count-rate for the PN in the $0.5-7.3 \mathrm{keV}$ band, averaged over the entire observation (see notes for the quiescent level of the sources with strong flares). $L_{\mathrm{X}}$ is estimated in the $0.3-10 \mathrm{keV}$ band from CR (see text), except where noted. A "y" in the column "var" means that the X-ray source was found to be variable according to the maximum likelihood algorithm described in Sect. 5. The 7th column is the designation of the infrared counterpart in the 2MASS catalogue; "offset" is the distance between the positions of the X-ray source and its IR counterpart; $J, H$ and $K_{\mathrm{s}}$ are the infrared magnitudes reported in the 2MASS catalogue. Sources in bold face are the most probable candidates on the basis of light curve and/or spectral analysis (Sect. 5).

\begin{tabular}{|c|c|c|c|c|c|c|c|c|c|c|}
\hline XEST id & $\begin{array}{l}\text { RAX } \\
\text { h m s }\end{array}$ & $\begin{array}{c}\operatorname{Dec} X \\
\operatorname{deg}^{\prime}{ }^{\prime \prime}\end{array}$ & $\begin{array}{l}\mathrm{CR} \\
\mathrm{cts} \mathrm{ks}^{-1}\end{array}$ & $\begin{array}{c}L_{\mathrm{X}} \\
10^{28} \mathrm{erg} \mathrm{s}^{-1}\end{array}$ & var & desig. 2MASS & $\begin{array}{l}\text { Offset } \\
\text { arcsec }\end{array}$ & $\begin{array}{c}J \\
\mathrm{mag}\end{array}$ & $\begin{array}{c}H \\
\mathrm{mag}\end{array}$ & $\begin{array}{c}K_{\mathrm{s}} \\
\mathrm{mag}\end{array}$ \\
\hline $27-021$ & 35336.36 & +31524.1 & $1.1 \pm 0.3$ & 0.72 & $\mathrm{y}$ & $03533637+3152044$ & 0.39 & 15.24 & 14.58 & 14.21 \\
\hline $27-022$ & 35337.41 & +32459.2 & $7.9 \pm 0.8$ & 5.6 & & $03533730+3204582$ & 1.56 & 8.38 & 7.91 & 7.78 \\
\hline 27-084 & 35410.70 & +314857.6 & $18.0 \pm 0.7^{a}$ & 8.4 & $\mathrm{y}$ & $03541064+3148573$ & 0.73 & 12.39 & 11.64 & 11.36 \\
\hline $27-130$ & 35440.39 & +315311.0 & $8.4 \pm 0.6$ & 3.6 & $\mathrm{y}$ & $03544037+3153107$ & 0.28 & 13.32 & 12.70 & 12.34 \\
\hline $06-006$ & 40349.93 & +262038.2 & $2.4 \pm 0.6$ & 1.0 & & $04034997+2620382$ & 0.63 & 13.28 & 12.66 & 12.34 \\
\hline $06-041$ & 40424.49 & +2611 12.9 & $17.1 \pm 1.1$ & 7.3 & & $04042449+2611119$ & 0.90 & 12.10 & 11.45 & 11.19 \\
\hline $06-045$ & 40427.62 & +26833.7 & $1.5 \pm 0.5$ & 0.63 & & $04042766+2608349$ & 1.42 & 14.63 & 14.03 & 13.50 \\
\hline $20-045$ & 41415.84 & +281818.8 & $1.0 \pm 0.2$ & 1.9 & & $04141588+2818181$ & 0.89 & 14.05 & 12.98 & 12.47 \\
\hline $20-063$ & 41442.90 & +28219.3 & $1.3 \pm 0.4$ & 4.3 & & $04144294+2821105$ & 1.40 & 14.26 & 12.77 & 12.07 \\
\hline $20-066$ & 41447.22 & +2835.5 & $7.1 \pm 0.8$ & 3.0 & & $04144739+2803055$ & 2.30 & 10.80 & 10.17 & 9.92 \\
\hline 20-071 & 41452.35 & +2860.5 & $159 \pm 3$ & $171^{b}$ & & $04145234+2805598$ & 0.68 & 9.53 & 8.21 & 7.71 \\
\hline $23-062$ & 4191.15 & +283710.8 & $1.4 \pm 0.4$ & 0.61 & & $04190125+2837101$ & 1.48 & 11.81 & 11.14 & 10.88 \\
\hline $23-065$ & 4196.96 & +282610.3 & $1.8 \pm 0.3$ & 5.3 & & $04190689+2826090$ & 1.50 & 14.90 & 13.57 & 12.88 \\
\hline $24-057$ & 4196.85 & +28268.8 & $2.7 \pm 0.5$ & 8.1 & & $=$ XEST-23-065 & & & & \\
\hline $16-045$ & 42039.12 & +271731.6 & $30.4 \pm 2.4$ & 13 & & $04203918+2717317$ & 0.86 & 10.50 & 9.86 & 9.56 \\
\hline $21-026$ & 42140.18 & +281421.6 & $2.1 \pm 0.4$ & 0.9 & & $04214013+2814224$ & 1.05 & 11.93 & 11.34 & 11.03 \\
\hline $11-035$ & 42143.59 & +264720.7 & $2.1 \pm 0.5$ & 0.9 & & $04214372+2647225$ & 2.59 & 9.61 & 8.98 & 8.83 \\
\hline $11-078$ & 42215.65 & +26576.5 & $9.4 \pm 0.5$ & 23 & $\mathrm{y}$ & $04221568+2657060$ & 0.60 & 13.81 & 12.62 & 12.03 \\
\hline $11-087$ & 42224.04 & +264625.6 & $13.4 \pm 1.1$ & 16 & & $04222404+2646258$ & 0.19 & 11.09 & 10.19 & 9.77 \\
\hline $21-059$ & 42225.63 & +281233.4 & $8.9 \pm 0.8$ & 6.1 & & $04222559+2812332$ & 0.40 & 9.26 & 8.75 & 8.62 \\
\hline $11-088$ & 42227.18 & +265951.1 & $2.1 \pm 0.3$ & 1.6 & & $04222718+2659512$ & 0.19 & 15.28 & 14.59 & 14.19 \\
\hline $21-073$ & 42248.77 & +282259.9 & $3.6 \pm 0.3$ & 2.7 & & $04224865+2823005$ & 1.58 & 14.51 & 13.81 & 13.45 \\
\hline $02-005$ & 42634.97 & +26816.2 & $2.9 \pm 0.6$ & 5.7 & & $04263497+2608161$ & 0.07 & 15.79 & 14.77 & 13.93 \\
\hline $02-069$ & 42758.65 & +26116.3 & $1.7 \pm 0.4$ & 0.71 & & $04275871+2611062$ & 0.82 & 10.67 & 10.00 & 9.85 \\
\hline $13-002$ & 42858.45 & +243649.3 & $5.2 \pm 1.2$ & 17 & & $04285844+2436492$ & 0.03 & 13.27 & 11.70 & 11.07 \\
\hline $13-010$ & 42936.07 & +243554.7 & $22.1 \pm 1.1$ & 68 & & $04293606+2435556$ & 1.00 & 10.78 & 9.39 & 8.66 \\
\hline 15-034 & 42936.17 & +263423.4 & $24.4 \pm 1.0$ & $10^{b}$ & $\mathrm{y}$ & $04293623+2634238$ & 0.90 & 11.56 & 10.94 & 10.65 \\
\hline $15-075$ & 43017.10 & +262228.3 & $24.6 \pm 2.5$ & 10 & & $04301702+2622264$ & 2.09 & 11.80 & 11.16 & 10.91 \\
\hline $14-034$ & 43025.21 & +26257.2 & $0.8 \pm 0.3$ & 0.35 & & $04302526+2602566$ & 0.89 & 11.06 & 10.46 & 10.24 \\
\hline $13-036$ & 43041.61 & +243043.0 & $3.6 \pm 0.9$ & 1.5 & & $04304153+2430416$ & 1.68 & 10.22 & 9.60 & 9.38 \\
\hline $19-002$ & 43146.26 & +255839.3 & $3.1 \pm 0.9$ & 2.0 & & $04314634+2558404$ & 1.65 & 13.33 & 12.62 & 12.31 \\
\hline $22-071$ & 43158.57 & +181842.1 & $2.2 \pm 0.3$ & 1.3 & & $04315860+1818408$ & 1.30 & 10.27 & 9.48 & 9.22 \\
\hline $22-111$ & 43226.80 & +181823.0 & $9.9 \pm 1.3$ & 7.5 & $\mathrm{y}$ & $04322689+1818230$ & 1.30 & 12.80 & 12.00 & 11.69 \\
\hline $22-114$ & 43229.40 & +181358.5 & $31.4 \pm 1.5$ & 34 & & $04322946+1814002$ & 1.93 & 14.46 & 13.69 & 13.17 \\
\hline $19-041$ & 43235.85 & +255222.6 & $1.1 \pm 0.2$ & 0.94 & & $04323605+2552225$ & 2.75 & 15.20 & 14.45 & 14.10 \\
\hline 03-028 & 43239.45 & +24274.6 & $16.6 \pm 1.0^{a}$ & 4.1 & $\mathrm{y}$ & $04323949+2427043$ & 0.62 & 14.53 & 12.96 & 12.30 \\
\hline 03-033 & 43259.20 & +243040.2 & $2.5 \pm 0.6$ & 5.7 & & $04325921+2430403$ & 0.25 & 16.48 & 15.27 & 14.66 \\
\hline 04-005 & 43259.12 & +243040.8 & $4.9 \pm 0.7$ & 11.3 & & $=$ XEST-03-033 & & & & \\
\hline $19-083$ & 43324.99 & +255925.7 & $6.3 \pm 0.7$ & 5.9 & $\mathrm{y}$ & $04332491+2559262$ & 1.13 & 14.38 & 13.61 & 13.24 \\
\hline $17-036$ & 43326.28 & +224529.2 & $24.2 \pm 1.1$ & 61 & & $04332621+2245293$ & 0.98 & 11.80 & 10.50 & 9.92 \\
\hline 17-043 & 43333.03 & +225252.7 & $20.4 \pm 0.9^{a}$ & 1.2 & $\mathrm{y}$ & $04333301+2252521$ & 0.57 & 15.40 & 14.80 & 14.40 \\
\hline 17-059 & 43352.50 & +225627.0 & $57.4 \pm 1.9$ & $40^{b}$ & & $04335252+2256269$ & 0.33 & 10.24 & 9.47 & 9.11 \\
\hline 04-060 & 43355.57 & +24251.1 & $1.3 \pm 0.3$ & 0.74 & & $04335562+2425016$ & 0.94 & 10.06 & 9.29 & 9.00 \\
\hline $18-059$ & 43433.11 & +26242.0 & $6.7 \pm 1.1$ & 2.9 & & $04343322+2602403$ & 2.25 & 11.18 & 10.54 & 10.28 \\
\hline $12-012$ & 43451.50 & +24443.9 & $3.3 \pm 0.8$ & 3.7 & & $04345164+2404426$ & 2.41 & 13.19 & 12.37 & 11.97 \\
\hline 08-003 & 43456.86 & +225835.8 & $331 \pm 6^{a}$ & 162 & $\mathrm{y}$ & $04345693+2258358$ & 1.03 & 10.47 & 9.59 & 9.27 \\
\hline 08-014 & 43513.23 & +225919.5 & $62.9 \pm 1.9$ & $30^{b}$ & $\mathrm{y}$ & $04351316+2259205$ & 1.36 & 11.13 & 10.53 & 10.25 \\
\hline 08-027 & 43536.53 & +2350.8 & $5.3 \pm 0.7$ & 8.3 & & $04353651+2304590$ & 1.69 & 16.19 & 15.17 & 14.72 \\
\hline 08-033 & 43542.05 & +225222.6 & $27.2 \pm 0.9$ & $38^{b}$ & & $04354203+2252226$ & 0.14 & 11.25 & 10.39 & 9.99 \\
\hline $09-023$ & 43542.00 & +225222.1 & $28.7 \pm 1.9$ & 29 & & $=$ XEST-08-033 & & & & \\
\hline 08-047 & 43552.07 & +22553.5 & $5.8 \pm 0.6$ & 9.5 & & $04355209+2255039$ & 0.58 & 11.31 & 10.23 & 9.81 \\
\hline
\end{tabular}


Table 1. continued.

\begin{tabular}{|c|c|c|c|c|c|c|c|c|c|c|}
\hline XEST id & $\begin{array}{c}\text { RAX } \\
\text { h m s }\end{array}$ & $\begin{array}{c}\text { Dec X } \\
\operatorname{deg} \prime^{\prime} \prime \prime\end{array}$ & $\begin{array}{l}\text { CR } \\
\text { cts ks }^{-1}\end{array}$ & $\begin{array}{c}L_{\mathrm{X}} \\
10^{28} \mathrm{erg} \mathrm{s}^{-1}\end{array}$ & var & desig. 2MASS & $\begin{array}{l}\text { Offset } \\
\text { arcsec }\end{array}$ & $\begin{array}{c}J \\
\text { mag }\end{array}$ & $\begin{array}{c}H \\
\text { mag }\end{array}$ & $\begin{array}{c}K_{\mathrm{s}} \\
\mathrm{mag}\end{array}$ \\
\hline 08-049 & 43552.91 & +225058.9 & $118 \pm 2$ & $115^{b}$ & $\mathrm{y}$ & $04355286+2250585$ & 0.63 & 10.99 & 10.11 & 9.75 \\
\hline 09-033 & 43552.85 & +225057.6 & $81 \pm 3$ & $68^{b}$ & & $=$ XEST-08-049 & & & & \\
\hline 09-042 & 43558.98 & +223835.3 & $269 \pm 3$ & $172^{b}$ & $\mathrm{y}$ & $04355892+2238353$ & 0.67 & 9.32 & 8.60 & 8.37 \\
\hline 05-027 & 4403.49 & +255355.4 & $109 \pm 5$ & $202^{b}$ & & $04400363+2553547$ & 2.10 & 9.81 & 8.48 & 7.91 \\
\hline 07-005 & 44039.12 & +25403.9 & $8.3 \pm 0.7^{a}$ & 4.4 & $\mathrm{y}$ & $04403912+2540024$ & 1.46 & 15.61 & 14.66 & 14.11 \\
\hline $10-016$ & 4423.85 & +251956.0 & $0.9 \pm 0.3$ & 1.5 & & $04420376+2519533$ & 2.86 & 15.07 & 14.04 & 13.55 \\
\hline $26-031$ & 45538.46 & +30 3145.4 & $0.8 \pm 0.2$ & 0.66 & & $04553844+3031465$ & 1.16 & 11.35 & 10.56 & 10.25 \\
\hline $26-052$ & 45548.21 & +303014.9 & $4.7 \pm 0.3$ & 2.0 & $\mathrm{y}$ & $04554820+3030160$ & 1.15 & 11.89 & 11.22 & 10.95 \\
\hline $26-062$ & 45555.99 & +303620.6 & $9.5 \pm 0.4$ & 8.6 & $\mathrm{y}$ & $04555605+3036209$ & 0.94 & 10.47 & 9.66 & 9.27 \\
\hline $26-135$ & 45629.47 & +303611.5 & $0.8 \pm 0.2$ & 1.0 & & $04562935+3036115$ & 1.51 & 15.28 & 14.48 & 13.93 \\
\hline
\end{tabular}

${ }^{a}$ Quiescent count-rates $\left(\mathrm{cts} \mathrm{ks}^{-1}\right.$ ) for sources with strong flares: $15.0 \pm 4.3$ (XEST-27-084), $1.2 \pm 0.7$ (XEST-03-028), $2.9 \pm 2.3$ (XEST-17-043), $180 \pm 40$ (XEST-08-003), $2.7 \pm 2.0$ (XEST-07-005). ${ }^{b}$ Obtained from spectral fitting of PN data (see Sect. 5).
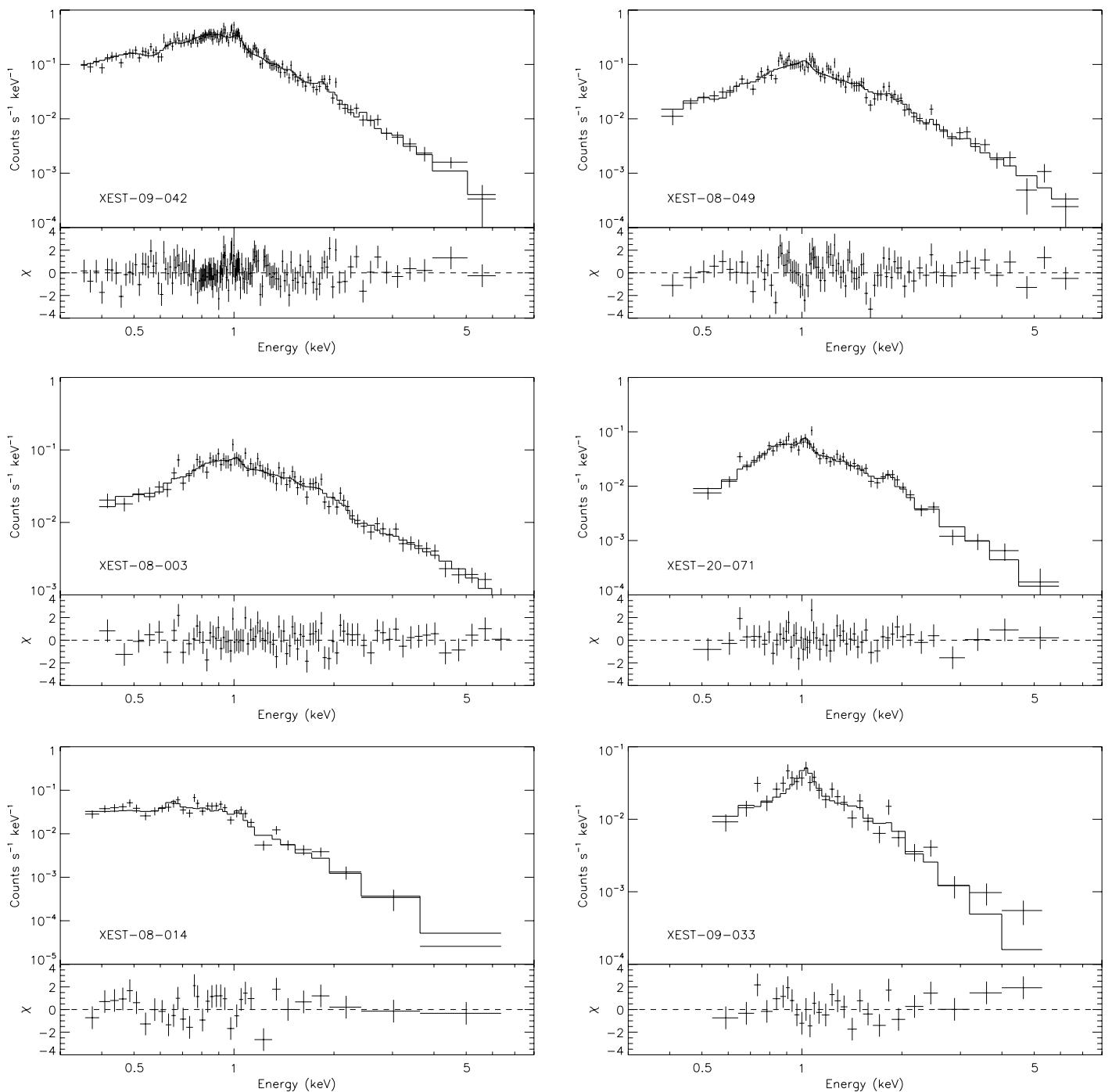

Fig. 7. EPIC PN spectra of the brightest candidate members (points) with the best-fit coronal models (see Table 2 for the parameters of the models). The lower panel in each plot shows the residuals in unit of $\chi$. 
L. Scelsi et al.: New pre-main sequence candidates in Taurus, Online Material $p 4$
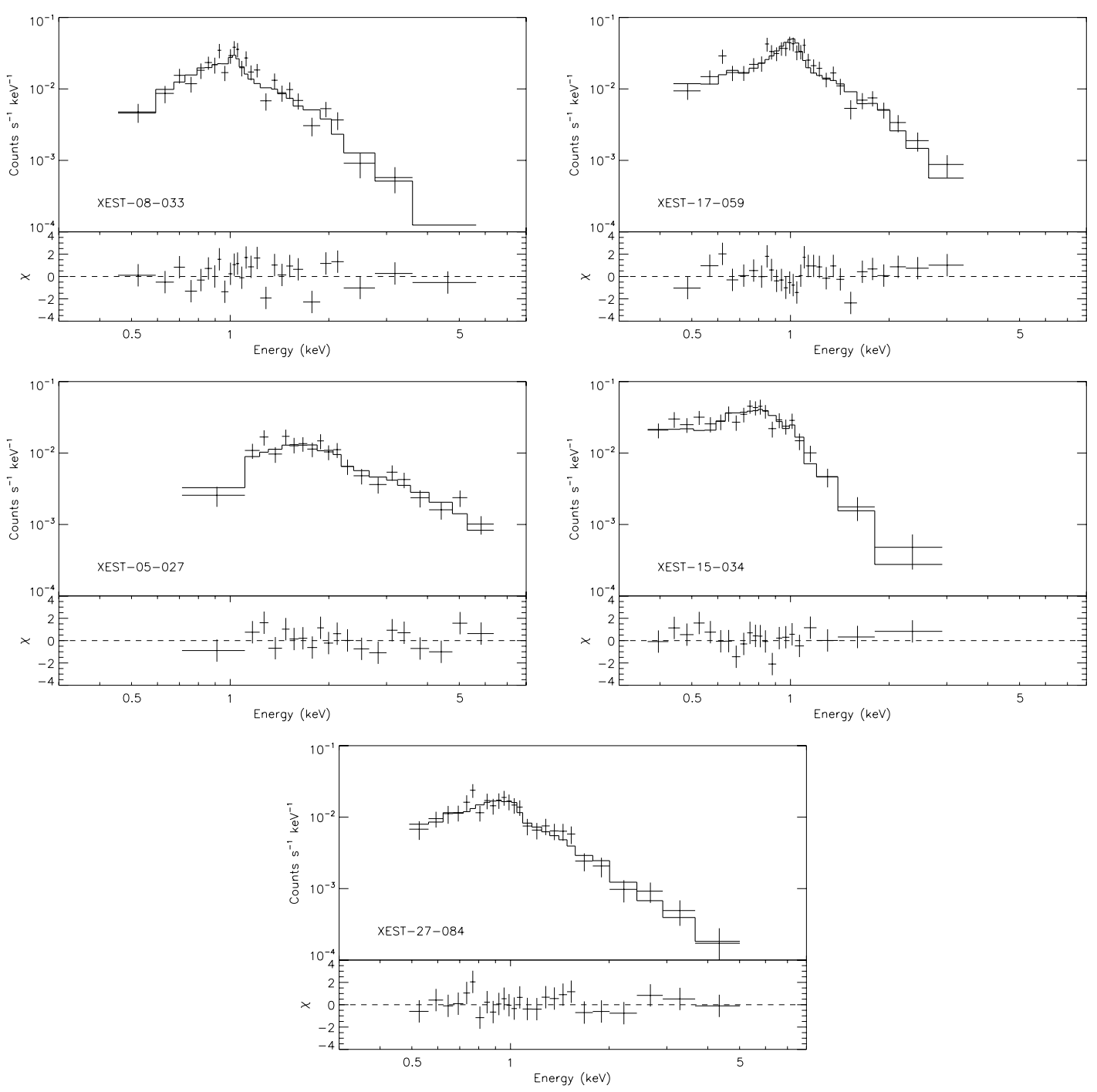

Fig. 7. continued. 